\title{
Kapitza resistance between superfluid helium and solid: role of the boundary
}

\author{
J. Amrit and A. Ramiere \\ Laboratoire d'Informatique pour la Mécanique et les Sciences de l'Ingénieur, LIMSI-CNRS UPR 3251, \\ Université Paris Sud, Rue John von Neumann, Orsay 91403, France \\ E-mail: jay.amrit@limsi.fr
}

Received March 14, 2013

\begin{abstract}
Kapitza resistance measurements conducted at $T>1 \mathrm{~K}$ on silicon and niobium single crystals in contact with helium demonstrate respectively the importance of atomic scale surface roughness and dislocations due to surface damage at the boundary. Two different experimental configurations were used.
\end{abstract}

PACS: 62.60.+v Acoustical properties of liquids;

63.20. $\mathrm{kp}$ Phonon-defect interactions;

67.80.bd Superfluidity in solid ${ }^{4} \mathrm{He}$, supersolid ${ }^{4} \mathrm{He}$.

Keywords: Kapitza thermal resistance, solid-helium interfaces.

\section{Introduction}

Almost three years after P.L. Kapitza discovered [1] superfluid helium in 1938, he observed an unusual jump in the temperature gradient across a copper/superfluid ${ }^{4} \mathrm{He}$ interface. This temperature jump $\Delta T$ is due to a thermal boundary resistance, often called the Kapitza resistance [2] and it is defined as $R_{K}=\Delta T /(\dot{Q} / A)$, where $(\dot{Q} / A)$ is the heat flux per unit area. Over the last decade the Kapitza resistance has gained further interest since it also plays a fundamental role in the field of nanothermal sciences which involves heat exchange at solid-solid micro nanointerfaces at room temperatures.

This paper deals with the original Kapitza resistance (KR) problem of a solid/superfluid ${ }^{4} \mathrm{He}$ interface for temperatures ranging from around $0.5 \mathrm{~K}$ to approximately $2 \mathrm{~K}$. To get an idea of the importance of KR we consider a parameter $L_{K i}=R_{K} K_{i}$ where $i=$ solid or $i=$ superfluid He. $K_{i}$ is the thermal conductivity and $L_{K i}$ corresponds to the length in medium $i$ whose thermal resistance (for the same cross-sectional area) is equivalent to the KR. For a $\mathrm{Cu} / \mathrm{He}$ interface at $T \sim 1 \mathrm{~K}, L_{\mathrm{K}, \mathrm{Cu}}$ is of the order of $\sim 10 \mathrm{~cm}$ and in helium, $L_{\mathrm{K}, \mathrm{He}}$ turns out to be of the order of $\sim 8000 \mathrm{~m}$. We recall that the KR occurs over atomic distances!

In summary, the acoustic mismatch theory (AMT) formulated by Khalatnikov [3] attributes the Kapitza temperature discontinuity to the very large discrepancies between the densities and the sound wave velocities of the two media. Phonon transmission from helium across the interface is therefore weak as it is confined within a narrow cone $\left(\sim 3^{\circ}\right)$; the latter defined by the conservation of frequency and the conservation of the parallel components of the momentum of phonons of each medium at the interface. These conditions also impose that the interface to be ideally smooth. The AMT prediction of the KR at solid/helium interfaces at $T \sim 1 \mathrm{~K}$ is almost two orders of magnitude greater than experimental values. And, experimental values generally tend to disagree with one another. However, experiments by Wyatt et al. [4] have clearly shown that phonon transmission is dominated by the background channel, that is, phonons incident from outside the critical value of the narrow cone. These puzzling features suggest that transmission mechanisms due to surface boundary non ideality (surface roughness, impurities, dislocations, surface orientation, change in He density close to the surface, defects ...) have to play an important role. And microscopic descriptions of scattering at the boundary are therefore necessary [5-8].

In this paper we present two recent experimental studies which highlight the impact of surface boundary effects at $T>1 \mathrm{~K}$. In the first experiment the pressure dependency of $\mathrm{KR}$ at a single-crystal silicon crystal/He interface is determined at $T \sim 1.82 \mathrm{~K}$. Surface roughness of the crystal is taken into account in analyzing these results. In the second experiment two surface states of single crystal niobium in contact with helium are studied for $1.5 \mathrm{~K}<T<2 \mathrm{~K}$. The presence of dislocations in the damaged layer near the boundary surface is considered to interpret our measured data. 


\section{Pressure dependency of the KR at a silicon/helium interface}

The acoustic properties of superfluid can be monitored by controlling its pressure. The aim in this study is to examine the influence of bulk superfluid on KR. First studies of the pressure dependency of KR were carried-out by Challis et al. [9]. Their experiments were conducted using copper. However, little is known about the purity and the surface state of copper in their experiment. The work here is conducted on a single silicon crystal (dielectric) of intrinsic purity and with a nanometer scale surface roughness state characterized by atomic force microscopy (AFM).

The experimental set-up is the same as that used in study [10] and it is shown in Fig. 1. The highly polished [111] surface of a single crystal silicon rod is anchored leak-tight into a thin walled stainless steel tube, which is filled with superfluid He. The temperature of the superfluid is controlled to within $1 \mathrm{mK}$ as a heat flux is directed along the (111) axis. The phonons backscattering in the crystal at the interface induce a temperature change detected by $\mathrm{RuO}_{2}$ thermometers placed at regular intervals of $12 \mathrm{~mm}$ along the crystal lateral surface. Detailed heat flow analysis in the cell is presented in Ref. 10 and the impact of heat losses on the measurements is well controlled. A heat flux change of $\Delta \dot{Q}$

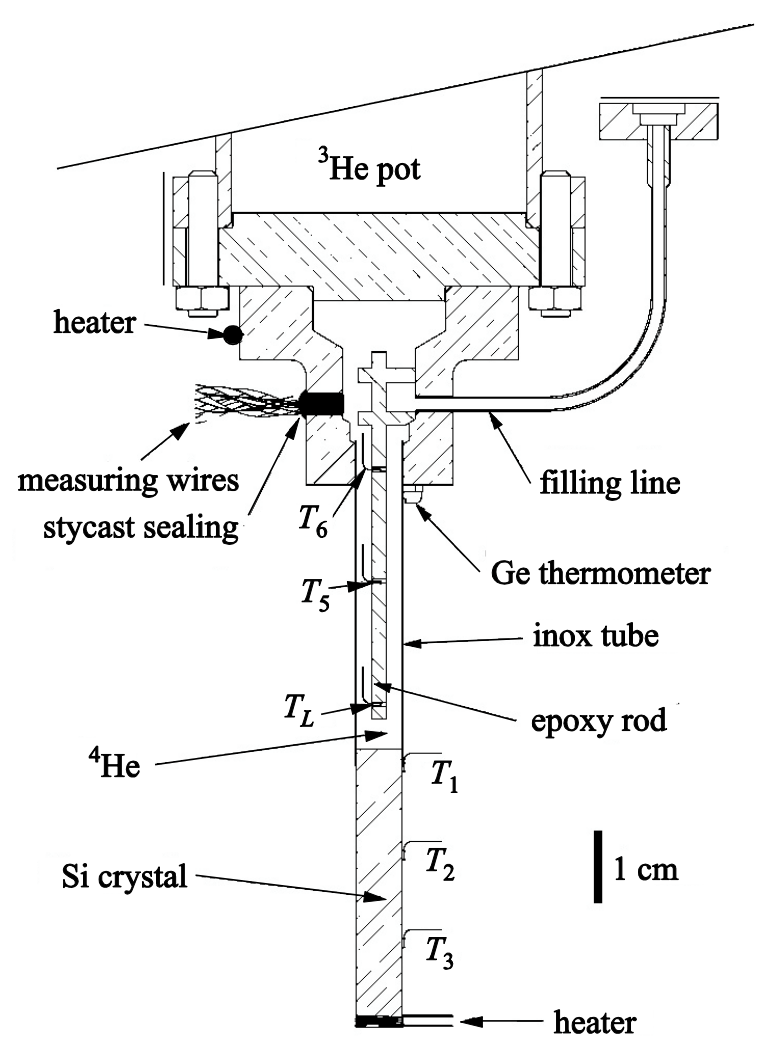

Fig. 1. Experimental cell configuration of a single crystal silicon rod in contact with helium. The Kapitza resistance is determined from measurements of temperature shifts in thermometer $T_{1}$. induces a temperature change in thermometer $T_{1}$ which is given by $\Delta T_{1}=\left(R_{K}+d / K\right) \Delta \dot{Q}$, where $d=2 \mathrm{~mm}$ is the distance between $T_{1}$ and the interface. In Ref. 10 it is shown that the measured thermal conductivity of the Si crystal has the following behavior $K_{\mathrm{Si}}=(1 / 3) C \mathrm{v}_{D} \ell$, where the specific heat $C=6.02 \cdot 10^{-7} T^{3}\left(\mathrm{~J} \cdot \mathrm{cm}^{-3} \cdot \mathrm{K}^{-1}\right)$, the Debye velocity $v_{D}=5.93 \cdot 10^{5} \mathrm{~cm} / \mathrm{s}$ and the mean free path $\ell=0.52 \mathrm{~cm}$. The pressure of the superfluid is directly monitored with help of a Bourdon manometer from SVP to $\sim 25$ bar, with a precision of $\sim 0.15$ bar.

The experimental results, shown in Fig. 2, display no change in the KR with pressure in the entire range from $\mathrm{SVP}$ to 25 bar. The KR has a constant value of $R_{K}=$ $=(5.22 \pm 0.2) \mathrm{cm}^{2} \cdot \mathrm{K} / \mathrm{W}$ at $T \sim 1.82 \mathrm{~K}$. If the KR were due (even partially) to a mismatch in the acoustic properties of each medium, then phonon transmission at a solid/superfluid interface would also depend on $\tau(P) \approx 4 Z_{L}(P) / Z_{S}$, where $Z_{L}$ and $Z_{S}$ are acoustic impedances of the superfluid and the solid. The ratio $Z_{L}(S V P) / Z_{L}(25$ bar $)=0.55$. The error bars in our measurements are $\leq 4 \%$. An $\sim 80 \%$ change in the phonon transmission would have been clearly detected in our experiment.

Our results strongly indicate that the influence of bulk properties of each medium play a negligible role on the transmission of phonons at this temperature. The transmission must therefore be entirely determined by one or more physical mechanism(s) taking place at the interface. The

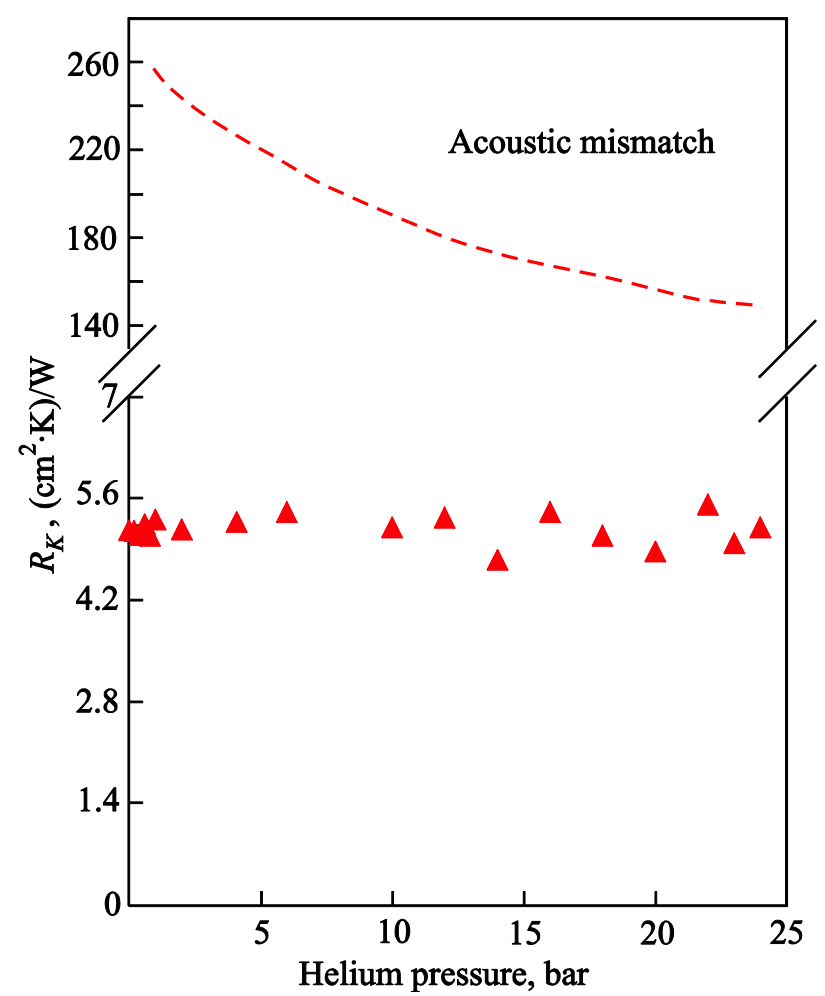

Fig. 2. Kapitza resistance measurements (full triangles) at silicon-helium interface as a function of pressure at $T \sim 1.82 \mathrm{~K}$. The dashed curve represents the acoustic mismatch theory prediction. 
interface morphology/structure is therefore of primary importance.

Here we give one possible explanation for the absence of pressure dependency of $\mathrm{KR}$ at $T \sim 1.82 \mathrm{~K}$. In our previous study [10] we interpreted the $\mathrm{KR}$ at SVP pressure from $0.4 \mathrm{~K}$ to $2 \mathrm{~K}$ with the aid of the Adamenko and Fuk's model (see Ref. 6). In this model the heat flux across the interface is "amplified" due to resonant phonon scattering when the phonon wavelength $\lambda$ becomes comparable to the surface roughness height $\sigma$. The model explains differences as large as two orders of magnitude between the experimental data and the AM theory.

Now the phonon wavelength in the superfluid is given by $\lambda=\left(h c_{L}(P)\right) /\left(3.8 k_{B} T\right)$. As the pressure is increased from SVP to $\sim 25$ bar the speed of sound $c_{L}(P)$ changes correspondingly from $239 \mathrm{~m} / \mathrm{s}$ to $365 \mathrm{~m} / \mathrm{s}$. Consequently, the wavelength varies from $\lambda \approx 3 / T$ at SVP to $\lambda \approx 4.6 / T$ at $\sim 25$ bar, with $\lambda$ in $\mathrm{nm}$ and $T$ in Kelvin. At $T=$ $=1.82 \mathrm{~K}, \lambda$ changes from $1.6 \mathrm{~nm}$ to $2.5 \mathrm{~nm}$ with pressure. As shown by AFM, our silicon surface has roughnesses which lie between $0.7 \mathrm{~nm}$ and $\sim 3 \mathrm{~nm}$ (see Ref. 10). With these values, it is clear that resonant scattering, determined by the ratio of $(\sigma / \lambda)$ which remains unchanged with pressure, is present at SVP as well as at $\sim 25$ bar and therefore no pressure effects are observable on phonon transmission at the boundary, as confirmed by our experimental data.

\section{Impact of surface impurities and dislocations on KR at single crystal $\mathrm{Nb} / \mathrm{He}$ interfaces}

In this study we focus on the impact of dislocations/strain and impurities present in the solid near surface boundary. During the cutting process of a solid material, defects, dislocations, strains and impurities can be created or introduced within a thickness of a few tens of $\mu \mathrm{m}$ from the surface, forming a "damaged layer". Depending on the nature of the polishing technique, the damaged layer maybe partially or fully removed. It is well-established that their presence can modify the thermal conductivity of solids due to phonon-dislocation interactions, for example. We have conducted systematic measurements on two sets of niobium samples labeled respectively "DL" for the sample with a damaged layer and "CP" for the sample that was chemically polished after the first series of measurements.

A schematic drawing of the cell is shown in Fig. 3. Two identical niobium samples (in the form of discs of $5 \mathrm{~cm}$ in diameter and $2 \mathrm{~mm}$ thick) are mounted on either sides of a cylinder to form a superfluid leak-tight cavity. The cavity contains a manganin-wire heater and an Allen Bradley $100 \Omega$ carbon resistor which serves as a thermometer. The cavity is placed in a temperature regulated superfluid bath and is filled via a $1.2 \mathrm{~m}$ long spiral filling-line open to the bath. Details on heat loss analysis through the cell are given in Ref. 11.

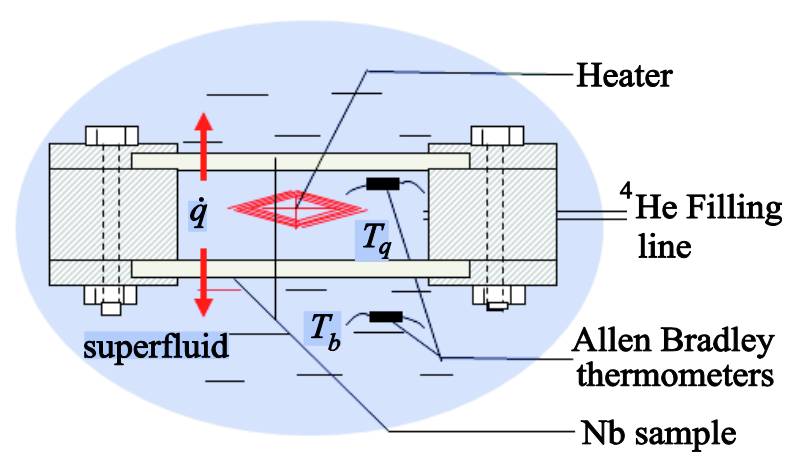

Fig. 3. Experimental cell showing Kapitza resistance determination from measurements conducted in two helium baths.

In the presence of a constant heat flux $\dot{q}$ dissipated in the cavity, only the temperature in the cavity increases from its initial temperature $T_{b}$ to $T_{q}$. The bath temperature remains controlled (within $1 \mathrm{mK}$ ) at $T_{b}$ throughout the measurement. The $\mathrm{KR}$ is then determined using: $\left(T_{q}-T_{b}\right)=\left[R_{K}+t /(2 K)\right] \dot{q}$, where $t$ is the sample thickness and $K$ is the thermal conductivity of the niobium sample. The factor 2 comes from the symmetry of the cell.

Our samples are single crystal niobium discs having a (111) crystallographic orientation. We determined the orientation by performing Electron Backscattering Diffusion (EBSD) measurements. The samples were cut by elec-

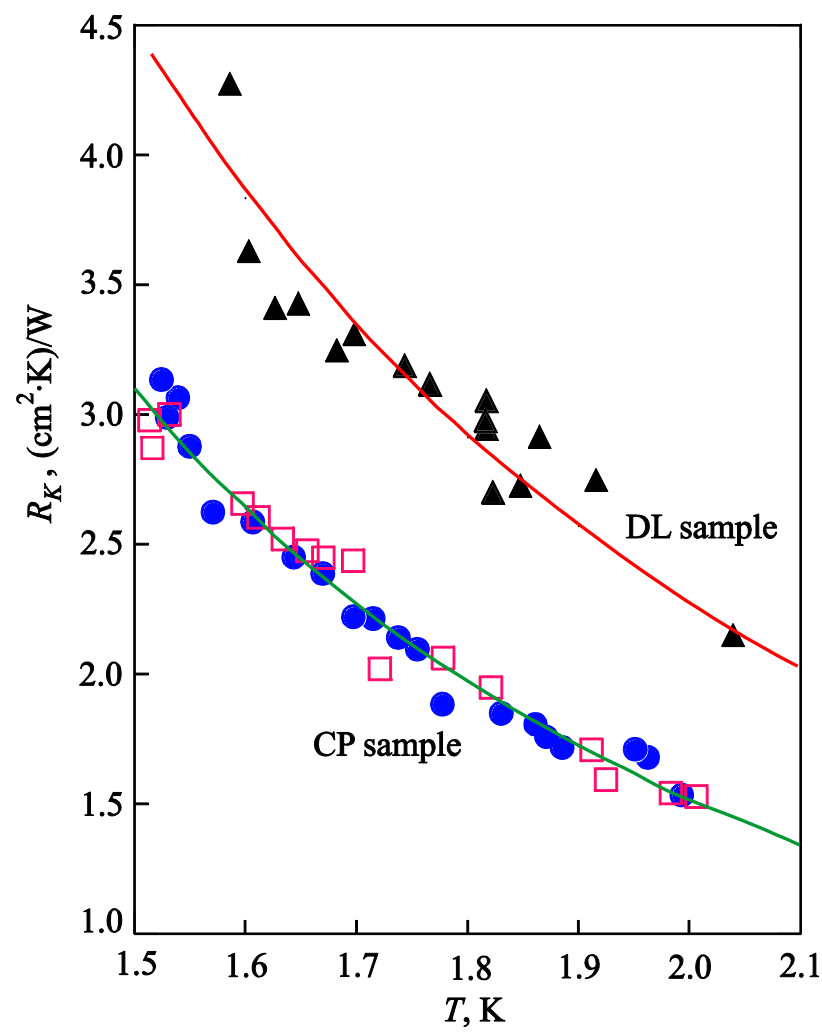

Fig. 4. Kapitza resistance $R_{K}$ for niobium samples with damaged layer ( $\Delta$ ) and after chemical polishing ( $\square$, for $2 \mathrm{~mm}$ thick samples) and (•, for $4 \mathrm{~mm}$ thick samples). The solid line fit to the DL sample take into account the density of dislocations (see text). 

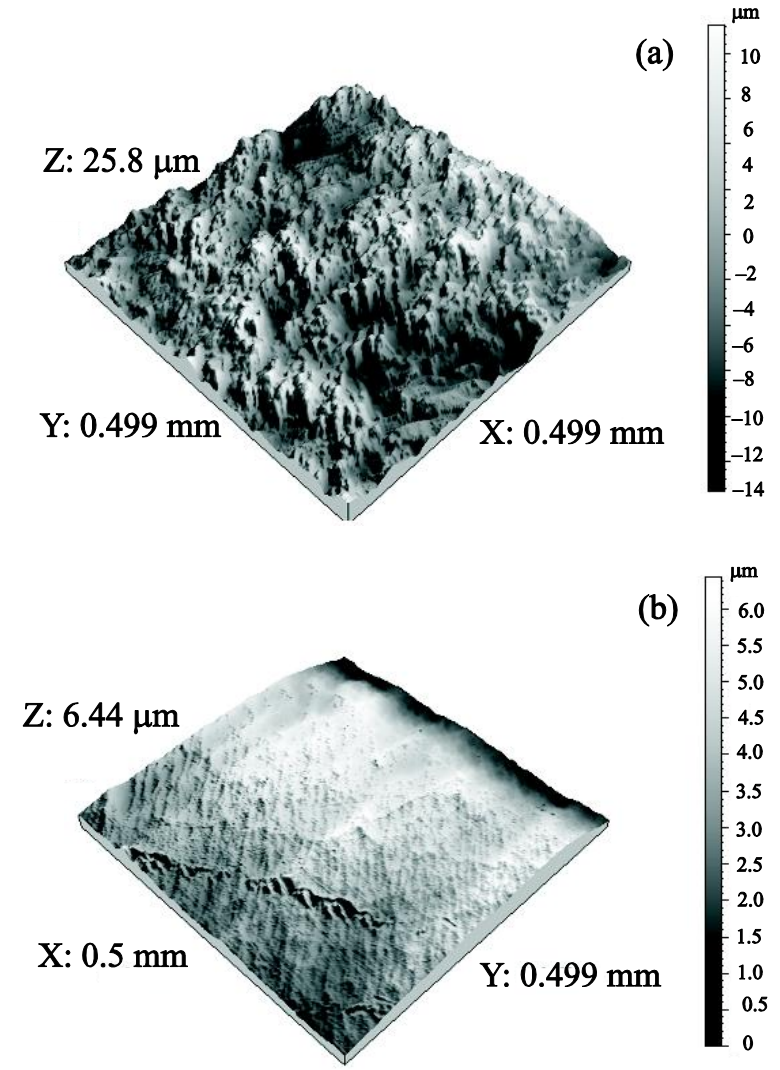

Fig. 5. Scanning electron microscope images of single crystal niobium (a) with damaged layer (b) after chemical polishing

trical discharge machining (EDM) from the same ingot which had a bulk purity characterized by a residual resistance ratio $(\mathrm{RRR})$ of $\sim 300$. They were cleaned in an ultrasonic bath of ultrapure ethanol. The $\mathrm{CP}$ sample was obtained by buffered chemical polishing (BCP) of DL samples.

Figure 4 shows measurements of the KR for each of these samples. The KR of the DL sample is greater than $\mathrm{KR}$ of the CP sample by 30 to $40 \%$. At first sight the results are somewhat unexpected for two reasons. The first reason is that the effective or "macroscopic" surface of the DL sample is greater than that of the CP sample as is clearly apparent from Figs. 5(a) and 5 (b). The rms surface roughness for our DL sample was $\sim 6 \mu \mathrm{m}$. This roughness is a result of the choice of the EDM cutting technique. The CP sample had a shiny surface with a roughness of $\sim 1 \mu \mathrm{m}$.

The second reason lies in the fact that the DL sample contains impurities. Its surface had a velvet-like texture due to an amorphous microcrystalline layer which contains impurities like $\mathrm{O}_{2}, \mathrm{Zn}$ and $\mathrm{Cu}$ particles within the uppermost layer of $\sim 1 \mu \mathrm{m}$. The impurity content is uniform along the surface and it decreases with depth to $\sim 1 \%$ over a thickness of $5 \mu \mathrm{m}$. Now, according to the theory of Khalatnikov and Adamenko [12], the presence of impurities of the solid surface should lead to a decrease of the KR, contrary to our experimental observations. They explain this decrease as follows. If the solid-helium is ideally smooth, then phonons incident from the liquid at angles greater than the critical angle $\theta_{c}=\arcsin \left(c_{L} / c_{S}\right)$ undergo total internal reflection, where $c_{L}$ and $c_{S}$ are respectively the sound velocities in the liquid and solid. At the same time in a narrow "skin" layer of the solid near the interface, there propagates an inhomogeneous surface wave, which like Rayleigh waves, do not transfer heat into the solid. However, the presence of impurities in the solid near the interface leads to the scattering of this surface wave, giving rise to energy transfer of this surface wave into the solid. This additional heat flux across the solid-helium interface leads to a decrease of the KR.

We suggest an alternate mechanism to explain our experimental observations. It is well-known that different cutting techniques induce strain and dislocations within a narrow layer $\ell_{d}$ (not exceeding a few $\mu \mathrm{m}$ in thickness) of the solid surface. The phonon-dislocation interaction which is now present modifies heat flow through the interface. To take into account this effect we note that the lattice thermal resistivity associated with the scattering of phonons by randomly distributed dislocations is calculated [13] for the case of niobium and is given by: $R_{d p}=3.05 \cdot 10^{-9} N_{d} / T^{2}$ $\left(\mathrm{cm}^{3} \cdot \mathrm{K}^{3}\right) / \mathrm{W}$, where $N_{d}$ is the density of dislocations per unit area within $\ell_{d}$ only. The thermal resistance $R_{d p} \ell_{d}$ is in series with the $\mathrm{KR}$ at the solid-helium interface and it is inherent only in the measurements of the DL sample. Indeed, besides a natural oxide layer of $\sim 5 \mathrm{~nm}$, the CP sample is free of the above mentioned impurities after $\sim 30 \mu \mathrm{m}$ of chemical polishing. To show the influence phonondislocation interactions, we fit the KR data of the DL sample with the following: $R_{K, D L}==R_{K, C P}+R_{d p} \ell_{d}$, where $R_{K, C P}=8.48 \cdot T^{-2.482} \mathrm{~cm}^{2} \cdot \mathrm{K} / \mathrm{W}$ describes the CP sample. The fit to the DL sample data as shown in Fig. 5 is done with $\ell_{d} \approx 1 \mu \mathrm{m}$ and with $N_{d}$ taken to be an adjustable parameter. The dislocation densities (in $\mathrm{cm}^{-2}$ ) fitting the data lie in a narrow range $8.8 \cdot 10^{12}<N_{d}<1.04 \cdot 10^{13}$. These values of the density of dislocations are very plausible for niobium as indicated in Ref. 13.

\section{Summary and conclusions}

The pressure dependency on KR was investigated in the first experiment conducted on a single silicon crystal having a polished surface with a surface roughness less than $3 \mathrm{~nm}$. The measurements showed no change in the KR for superfluid helium pressures from SVP to $\sim 23$ bar at $\sim 1.82 \mathrm{~K}$. We argued that this can be explained on the basis of the Adamenko and Fuk's model which introduces a mechanism of resonant phonon scattering when thermal phonons become comparable to nanometer-scale surface roughness. As showed in Ref. 10, this mechanism can also explain the discrepancy of a factor of $\sim 100$ between the measurements of $\mathrm{KR}$ as a function of temperature and the AMT.

The hypothesis that one (or more) solid layer(s) of helium at the interface adapts the impedance of the solid to 
that of the superfluid was examined by Challis et al. [9] Now, in this scenario, increasing the pressure would lead to an increase in the thickness of the solid He layers. One might therefore expect a gradual change in KR upon complete solidification of helium. We are currently investigating this aspect.

In the second experiment conducted on single crystal niobium samples, we examined the effect of a damaged layer (DL) on KR. We argued that the phonon-dislocation interactions are preponderant compared to the Khalatnikov and Adamenko dissipation mechanism due to the presence of impurities. We simply considered the thermal resistance due to phonon-dislocation interactions within a narrow layer at the interface to be in series with the KR at the niobiumhelium interface. There is perhaps need for more refined theoretical models to take into account these features. There is also need for more experiments on samples with well characterized density of dislocations. We believe that this feature may perhaps also contribute in understanding the large disparity in the multitude of experimental data [14], especially in the earlier measurements of the Kapitza resistance.

Experimental work on silicon crystal was conducted at the Institute de Physique Nucléaire d'Orsay. One of us (JA) expresses his thanks to I. Adamenko for fruitful discussions. We thank Claire Z. Antoine for doing the scanning electron microscope surface profiles. Part of this work was conducted under the grant PresUniverSud No. 2011-13.
1. The discovery of superfluidity was published simultaneously by P.L. Kapitza, and by J.F. Allen and A.D. Misener in the same issue of Nature in 1938.

2. P.L. Kapitza, Sov. Phys. JETP 11, 1 (1941).

3. I.M. Khalatnikov, An Introduction to the Theory of Superfluidity, Addison-Wesley, New York (1988).

4. A.F.G. Wyatt and G.N. Crisp, J. Phys. Colloq. 39, C6-244 (1978).

5. F.W. Sheard, R.M. Bowley, and G.A. Toombs, Phys. Rev. A 8, 3135 (1973).

6. I.N. Adamenko and I.M. Fuks, Sov. Phys. JETP 32, 1123 (1971).

7. I.N. Adamenko, K.E. Nemchenko, and I.V. Tanatarov, Phys. Rev. B 77, 174510 (2008).

8. I.N. Adamenko and K.E. Nemchenko, J. Low. Temp. Phys. doi 10.1007/s10909-012-0754-9 (2012).

9. L.J. Challis, K. Dransfeld, and J. Wilks, Proc. R. Soc. London 260, 31 (1961).

10. J. Amrit, Phys. Rev. B 81054303 (2010).

11. J. Amrit and M.X. François, J. Low. Temp. Phys. 119, 27 (2000).

12. I.M. Khalatnikov and I.N. Adamenko, Sov. Phys. JETP 36, 391 (1973).

13. W. Wasserbäch, Philos. Mag. A 38401 (1978).

14. E.T. Swartz and R.O. Pohl, Rev. Mod. Phys. 61, 605 (1989). 